\title{
GMR
}

\section{Tetradecyl 2,3-dihydroxybenzoate promotes functional recovery after spinal cord injury in adult rats}

\author{
Y.M. Ding ${ }^{1}$, J.Y. Zhu ${ }^{1}$, S.J. Zhang ${ }^{1}$, S.S. Zhang ${ }^{1}$, C. Wang ${ }^{1}$, L.L. Wang ${ }^{2}$ \\ and $X$. Zhang ${ }^{2}$ \\ ${ }^{1}$ Department of Clinical Medicine, School of Medicine, \\ Zhejiang University City College, Hangzhou, Zhejiang, China \\ ${ }^{2}$ Department of Basic Medical Science, \\ Zhejiang University School of Medicine, Hangzhou, Zhejiang, China \\ Corresponding author: X. Zhang \\ E-mail: xiongzhang@zju.edu.cn \\ Genet. Mol. Res. 15 (2): gmr.15028653 \\ Received March 23, 2016 \\ Accepted April 11, 2016 \\ Published May 13, 2016 \\ DOI http://dx.doi.org/10.4238/gmr.15028653
}

\begin{abstract}
Tetradecyl 2,3-dihydroxybenzoate (ABG001) is a small molecule separated from gentian extract that has a similar effect to nerve growth factor. It is not clear whether it can promote functional recovery in animals suffering from a central nervous system injury. In order to explore the role of $\mathrm{ABG} 001$ in restoration of tissue structure and motor function of rats with spinal cord injury (SCI), ABG001 $(0.4 \mathrm{mg} / \mathrm{kg})$ was administered intraperitoneally. Subsequently, behavioral assessments and morphological studies were performed to detect recovery of hind limb motor function and neuroregeneration. The results showed that compared with DMSO group, the rats in the ABG treatment group had better performance in $\mathrm{BBB}$ score and grip strength test $(\mathrm{P}<0.05)$, the area of necrosis was smaller $(\mathrm{P}<0.05)$, GFAP expression was significantly reduced $(\mathrm{P}<0.01)$, and Map-2 expression was significantly increased $(\mathrm{P}<0.01)$. Additionally, after ABG treatment, the number of fluorogold positive cells transported reversely to red nucleus increased $(\mathrm{P}<0.05)$.
\end{abstract}


The results suggest that $\mathrm{ABG} 001$ can promote recovery of hind limb motor function in rats with SCI, which may be related to its functions of inhibiting glial cell proliferation and promoting neuroregeneration.

Key words: Spinal cord injury; Tetradecyl 2,3-dihydroxybenzoate; Nerve growth factor; Neural regeneration

\section{INTRODUCTION}

Spinal cord injury (SCI) is a transverse injury of the spinal cord caused by various pathogenic factors such as trauma, inflammation, or tumors that produce spinal cord dysfunction below the level of the lesion. This not only brings serious physical and psychological damage to patients, but also causes a tremendous economic burden to society. According to statistics, SCI affects approximately 2.5 million people worldwide, and more than 130,000 new injuries are reported annually (Thuret et al., 2006).

Neurotrophic factor has good prospects in the treatment of SCI, but many problems exist with this treatment, such as low oral bioavailability, low permeability of the blood-brain barrier, and high manufacturing cost. Recently, some natural small molecules with similar structure and function to neurotrophic factor have been discovered. These molecules can overcome the above problems and may have a role in the treatment of spinal cord injury. For example, Gao et al. $(2010 \mathrm{a}, \mathrm{b})$ purified a novel group of compounds with similar structure to neurotrophic factor from Yunnan gentian and found that tetradecyl 2, 3-dihydroxybenzoate (ABG001) has the same function as nerve growth factor (NGF) in promoting growth and extending the PC12 cell neurite. This molecule is more stable than NGF and passes more easily through the blood-brain barrier (Gao et al., 2010a,b). Additionally, there is a certain structureactivity relationship between the structure of these compounds and their effect of promoting neurite growth (Luo et al., 2011). Subsequently, Zhou et al. (2014) administered an oral dose of ABG001 to adult mice for $5 \mathrm{~d}$ and found that it can not only dose-dependently enhance the survival of newborn neurons in dentate gyrus and the neurite growth, but can also improve the spatial cognitive performance of the dementia mice after intracerebroventricular injection of $\mathrm{A} b_{25-35}$ (Zhou et al., 2014). It therefore appears that ABG001 has a neuroprotective function and promotes neurite growth. However, the effectiveness of ABG001 for axonal regeneration has not been reported. Based on the above research results, the function of ABG001 in axonal regeneration and motor function recovery of rats with spinal cord injury was studied in this experiment.

\section{MATERIAL AND METHODS}

\section{Animals}

A total of 36 adult (200-250 g) female Sprague-Dawley rats were obtained from the Experimental Animal Centre of Zhejiang University (Hangzhou, China). Rats were housed under controlled temperature and lighting, with free access to food and water. All procedures were approved by the Animal Ethics Committee of Zhejiang University and were carried out in accordance with institutional guidelines. The rats were randomly divided into the sham group, $\mathrm{SCI}+\mathrm{ABG}$ group, and $\mathrm{SCI}+\mathrm{DMSO}$ group $(\mathrm{N}=12$ in each group). 


\section{Spinal cord contusion injury}

Rats were anesthetized with an intraperitoneal (i.p.) injection of $10 \%$ chloral hydrate ( $3 \mathrm{~mL} / \mathrm{kg}$ body weight). The vertebral column was exposed, and a T10 laminectomy was performed. Subsequently, a contusion injury was produced using a weight-drop device, as described previously (Liu et al., 2013). Rats in the sham group received a laminectomy but no SCI. After surgery, rats were administered normal saline by subcutaneous injection to replace lost fluids. Manual bladder expression was performed twice a day. To prevent infections, penicillin ( $100 \mathrm{mg} /$ day) was administered twice daily for 3 days post-injury. Twenty-four hours after the operation, rats in the $\mathrm{SCI}+\mathrm{ABG}$ group were treated with i.p. injection of $\mathrm{ABG}$ $(0.4 \mathrm{mg} / \mathrm{kg})$ every other day for 3 weeks, while rats of the SCI + DMSO group received the same quantity of DMSO.

\section{Behavioral testing}

The Basso, Beattie, and Bresnahan (BBB) locomotor rating scale and hind limb grip strength testing were performed by observers blinded to the treatments. The BBB scale was performed on each animal before SCI and on the first day after SCI to confirm the successful contusion (Basso 2004). Subsequently, the scale was used again on day 3, 6, 9, 12, 15, 18, and 21 after SCI. On each testing day, when BBB scale was finished, hind limb grip strengths were separately assessed using a grip strength meter (Bioseb, Chaville, France) according to a previously described method (Lepore et al., 2008). The average force by two hind limbs was calculated from five trials for each time point.

\section{Tissue processing}

At the end of the third week post-injury, six rats were randomly selected from each group and perfused transcardially with $0.1 \mathrm{M}$ phosphate buffer, followed by ice-cold $4 \%$ paraformaldehyde under anesthesia. The thoracic regions of the spinal cord were harvested for histological examination. The dissected spinal cord tissues were post-fixed for $4 \mathrm{~h}$ in $4 \%$ paraformaldehyde and soaked for $24 \mathrm{~h}$ in $10 \%$ sucrose, followed by $24 \mathrm{~h}$ in $20 \%$ and $24 \mathrm{~h}$ in $30 \%$ sucrose. Tissue surrounding the lesion site was embedded in OCT (SAKURA, Japan), fast-frozen with dry ice, and stored at $-80^{\circ} \mathrm{C}$ until processing. Spinal cord tissue blocks were cut into 30-mm longitudinal sections using a cryostat (Thermo, USA). Sections were collected on glass slides and stored at $-20^{\circ} \mathrm{C}$ until analysis.

\section{Histological analysis}

For general histological examination, sections of the spinal cord were stained by a Hematoxylin Eosin (HE) staining kit (Beyotime, China). The operation was performed according to the instructions of the kit. Briefly, sections were rinsed in distilled water and stained in hematoxylin for $5 \mathrm{~min}$. After washing, sections were differentiated in $1 \%$ acidalcohol for $30 \mathrm{~s}$ and were incubated with eosin for $30 \mathrm{~s}$. After dehydration with alcohol, sections were covered with xylene-based mounting medium and imaged using a Nikon microscope. Image-Pro Plus (Media Cybernetics, USA) was used to measure the necrotic areas within the injured spinal cord. 
For immunohistochemistry, sections of the spinal cord were subjected to glial fibrillary acidic protein (GFAP)/microtubule associated protein-2 (Map-2) double immunostaining, as previously described (Ding et al., 2013). Briefly, slices were dried at room temperature, rinsed with $0.01 \mathrm{M}$ phosphate buffer solution (PBS) for $30 \mathrm{~min}$, and blocked in $10 \%$ normal goat serum in PBS at room temperature for $2 \mathrm{~h}$ to prevent nonspecific staining. Then, sections were incubated with the primary antibodies such as mouse anti-GFAP polyclonal antibody (1:1000, CST, USA) and rabbit anti-Map-2 polyclonal antibody (1:100, Santa Cruz, USA) overnight at $4{ }^{\circ} \mathrm{C}$. The following day, sections were washed three times with PBS. Fluorescein isothiocyanate (FITC)-labeled goat anti-mouse $\operatorname{IgG}$ (1:200; Jackson Immuno Research Lab, USA) and Cy3-labeled goat anti-rabbit IgG (1:200; Jackson Immuno Research Lab) were incubated at room temperature for $2 \mathrm{~h}$, followed by a wash with PBS. The specimens were mounted onto gelatin-coated glass microscope slides, air dried, coverslipped in a glycerolbased mounting medium, and observed under a confocal microscope (FV1000, Olympus, Japan). The nuclei were stained by 4', 6'-diamidino-2-phenylindole (DAPI). The relative intensity of GFAP and Map-2 was measured with Image-Pro Plus. The value of the SCI + DMSO group was selected as a reference and the fluorescence intensity of the SCI + ABG group was compared to derive the relative fluorescence intensity.

\section{Fluorogold labeling}

At 6 weeks after contusion, the remaining rats from each group were anesthetized as described above and the T10 injury site was exposed again. Fluorogold (FG, $1 \mu \mathrm{L}$, Sigma, USA) was injected into the spinal cord at four points $(0.5 \mathrm{~mm}$ depth, $0.3 \mathrm{~mm}$ and $0.6 \mathrm{~mm}$ bilaterally to the midline, $0.25 \mu \mathrm{L}$ each), about $5 \mathrm{~mm}$ caudal to the contusion site. All injections were performed by the same researcher, to maximize comparability between animals. Muscles were sutured in layers and the skin was closed. Animals were returned to their cages for an additional week.

\section{Fluorogold counting}

One week after the injection of FG, the rats were sacrificed and perfused under anesthesia as described above. The brain was dissected and post-fixed, and then cut into 50$\mathrm{mm}$ sections. Sections were photographed using a confocal microscope (FV1000). FG-labeled neurons were counted according to a previously described method (Barbour et al., 2013). Briefly, brain sections were separately mounted on glass slides. The rubrospinal FG-positive neurons in the area of the red nucleus were counted and averaged. Six animals from each experimental group were used for this experiment and five brain sections from each animal were analyzed.

\section{Statistics}

Data were expressed as means \pm standard deviation (SD) and processed with SPSS 12.0 software. In the analysis of the behavioral testing scores, groups were compared using the twoway analysis of variance (ANOVA), followed by Dunnett's method of multiple comparisons versus control groups. For FG labeling of red nucleus (RN) neurons, experimental groups were compared using a one-way ANOVA, followed by the Tukey test for multiple comparison 
procedures between the experimental and control groups. A P value of less than 0.05 was considered statistically significant.

\section{RESULTS}

\section{ABG001 improves locomotor recovery after spinal cord injury in rats}

To investigate the possible functional role of ABG001, rats with SCI were treated with ABG001 (0.4 mg/kg, i.p. ) every other day for 3 consecutive weeks, and thereafter tested for locomotor function. As shown in Figure 1, the BBB scores and the hind limb grip strength of rats subjected to SCI were significantly lower than those of sham rats, with minimal values on the next day after injury $(\mathrm{P}<0.05)$. From day 9 post-injury onward, rats treated with $A B G$ showed improved locomotor behavior compared to rats treated with DMSO $(\mathrm{P}<0.05$, Figure $1 \mathrm{~A})$. Additionally, the repeated $\mathrm{ABG}$ treatments also enhanced the hind limb grip strength of the rats $(\mathrm{P}<0.05$, Figure 1B).

\section{A}

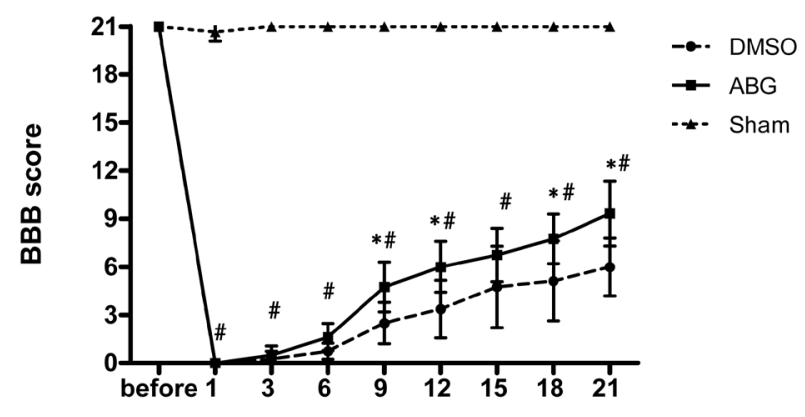

B

days post-injury

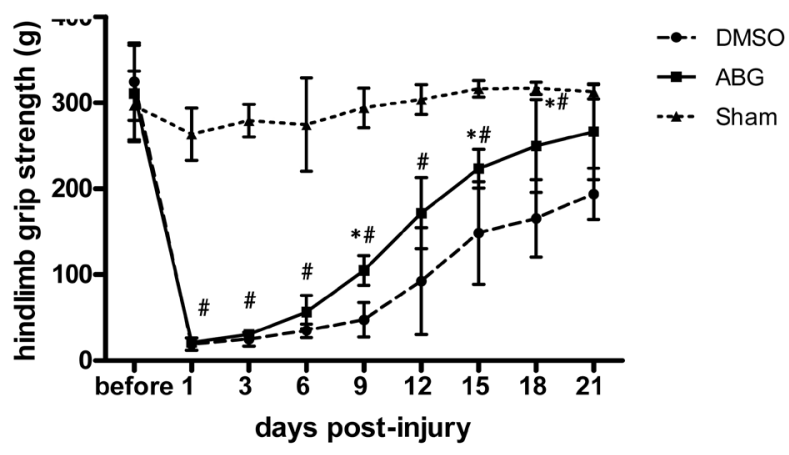

Figure 1. Locomotor function assessed using the BBB score and hind limb grip strength test. Within 3 weeks of the initial contusion of the spinal cord, the BBB scores (A) and the hind limb grip strength (B) of the SCI rats significantly decreased compared with the sham group $\left({ }^{\#} \mathrm{P}<0.05\right)$. Following treatment with ABG001 for 3 consecutive weeks, the use of the hind limbs increased gradually. Twelve days after injury, both the BBB scores and the hind limb grip strength were significantly greater in ABG-treated rats than in DMSO control rats $(* \mathrm{P}<$ $0.05, \mathrm{~N}=12$ ). Data are reported as means $\pm \mathrm{SD}$. Comparisons were conducted by two-way ANOVA followed by the Bonferroni multiple-comparison test. 


\section{ABG001 reduces the necrotic area in the spinal cord injury zone}

At the end of the third week after injury, HE staining was carried out to evaluate the morphological change and actual area of necrosis. In the DMSO group, HE-stained sections showed that the structure of the spinal cord was severely damaged. Neurons in gray matter were dissolved. Inflammatory infiltration was observed and a hemorrhage occurred at the lesion epicenter after contusion (Figure 2A). While the spinal cord also appeared to have visible structural damage in the ABG group, inflammatory cell infiltration, neuronal loss, and disintegration were milder in the ABG group than in the DMSO group (Figure 2B). Additionally, the necrotic area of spinal cord injury zone in the ABG group was significantly smaller than in the DMSO group $(\mathrm{P}<0.05$, Figure $2 \mathrm{C})$.

A

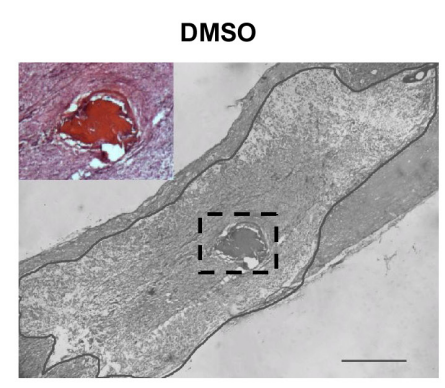

B

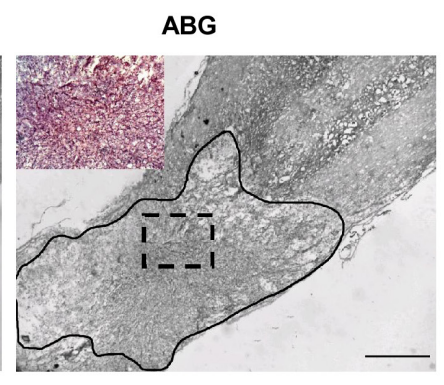

C

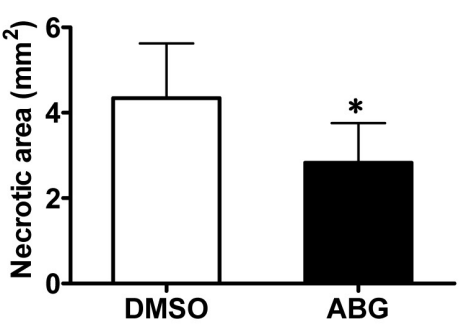

Figure 2. Hematoxylin-eosin staining in the spinal cord damage zone following treatment with ABG. A. Representative images of spinal cord injury zone in DMSO group. B. Representative images of spinal cord injury zone in ABG group. Color pictures show the higher magnification of the boxes in the pictures. Necrotic area is represented by the region surrounded by the black solid line. Scale bars, $100 \mathrm{~mm}$. C. Quantification of necrotic area of spinal cord injury zone. The area of necrosis in the ABG group significantly reduced compared with the DMSO group. Asterisk indicates a significant difference compared with DMSO group $(* \mathrm{P}<0.05, \mathrm{~N}=3)$. Data are expressed as means $\pm \mathrm{SD}$. Intergroup comparisons were conducted by one-way ANOVA followed by the post hoc test.

\section{ABG001 inhibits glial scar formation and protects neurons in injured spinal cord}

Immunohistochemistry showed that there were different amounts of immunoreactivity for GFAP and MAP-2 in the spinal cord injury zone (Figure 3 ). In the DMSO group, strong GFAP immunoreactivity was detected in the damage zone, indicating the formation of a glial scar at the edge, while a very small amount of Map-2 expression was observed within the damage zone. In the ABG001-treated group, however, GFAP immunoreactivity decreased significantly, while Map-2 expression showed an increased distribution in the damage zone. The relative intensity of GFAP fluorescence in the ABG group $(2.21 \pm 0.53)$ was significantly lower than in the DMSO group $(5.34 \pm 1.43, \mathrm{P}<$ 0.01 ), whereas the intensity of Map-2 fluorescence in the ABG group was much higher than in the DMSO group $(4.42 \pm 1.11$ vs $1.74 \pm 0.53, \mathrm{P}<0.01)$. Double immunostaining for GFAP and Map-2 showed that ABG significantly inhibited the formation of a glial scar and exhibited a possible neuroprotective function. 

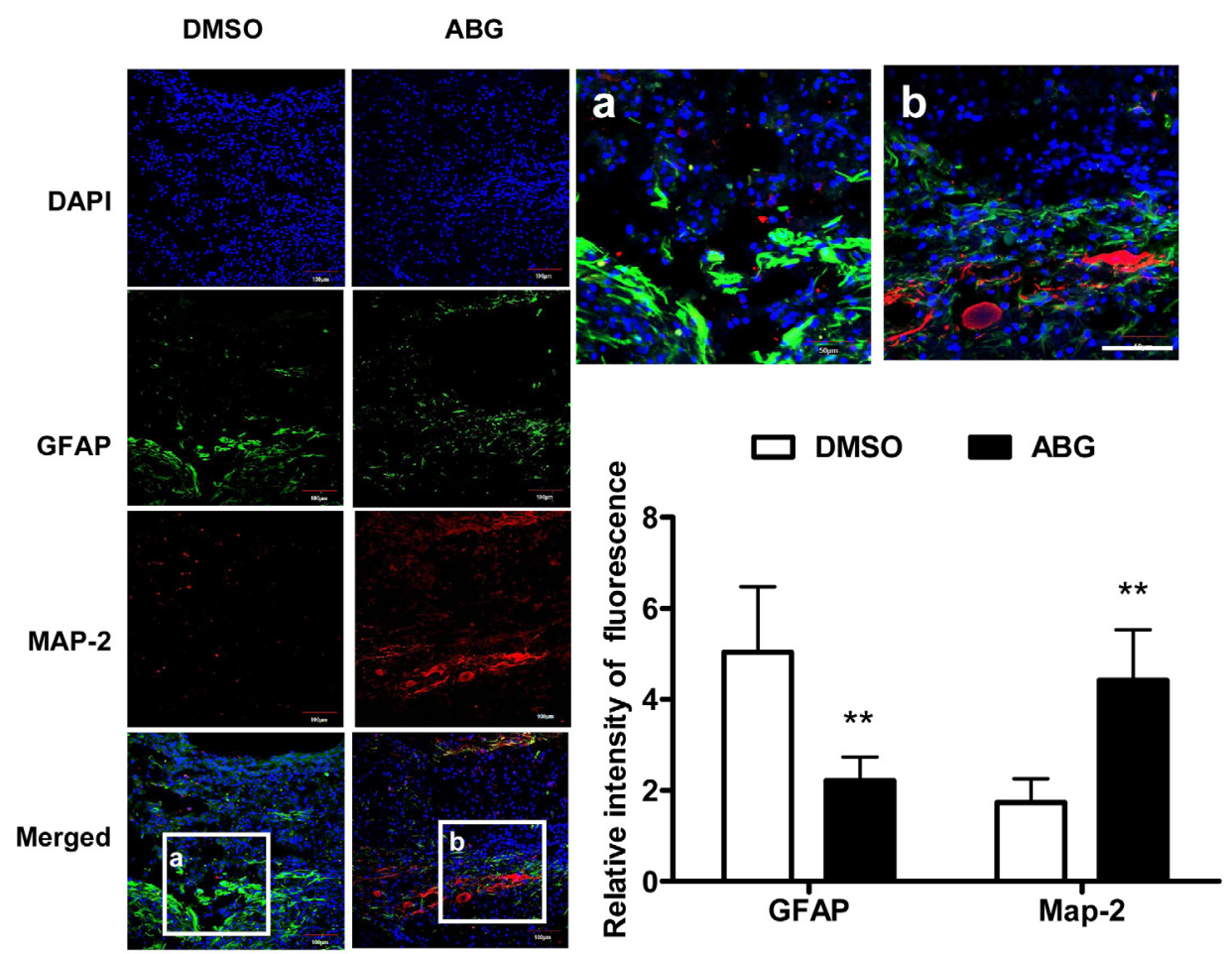

Figure 3. Double immunostaining for GFAP and Map-2 of injured spinal cord treated with ABG under a fluorescence microscope. Twenty-one days after spinal cord injury, GFAP/Map-2 double labeling was performed to evaluate the expression of Map-2. Scale bar, $100 \mathrm{~mm}$. Map-2, specific marker of neurons, is shown in red; GFAP, labeled glial scar, is shown in green. A. B. Higher magnification of the boxes in the pictures accordingly. Scale bar, $40 \mathrm{~mm}$. The expression of GFAP in the ABG group was less than in the DMSO groups and the Map-2 expression increased in the $\mathrm{ABG}$ group $(* * \mathrm{P}<0.01, \mathrm{~N}=3$ ). Data are reported as means $\pm \mathrm{SD}$. Intergroup comparisons were conducted by one-way ANOVA followed by the post hoc test.

\section{ABG001 increases the number of retrogradely FG-labeled red nucleus neurons}

To quantify the rubrospinal tract regeneration, fluorogold was injected bilaterally 5 mm caudal to the contusion site in the spinal cord. The number of FG-labeled red nucleus neurons was counted in all three groups of animals (Figure 4). In sham animals, about 100 neurons were labeled in the $\mathrm{RN}$ in each section. After T10 contusion, only 30 cells were labeled in the $\mathrm{RN}(\mathrm{P}<0.05)$ in the DMSO group. With ABG treatment, about 70 cells were retrogradely labeled in the $\mathrm{RN}$; this number was significantly different from that of DMSO controls $(\mathrm{P}<0.05)$. Thus, ABG may have provided a permissive environment that allowed a very small percentage of axotomized neurons to regenerate in the caudal spinal cord.

\section{DISCUSSION}

Extensive research has been conducted on the treatment of spinal cord injury (Kim et al., 2011; Hu et al., 2013). Axonal regeneration and the formation of functional synapses 

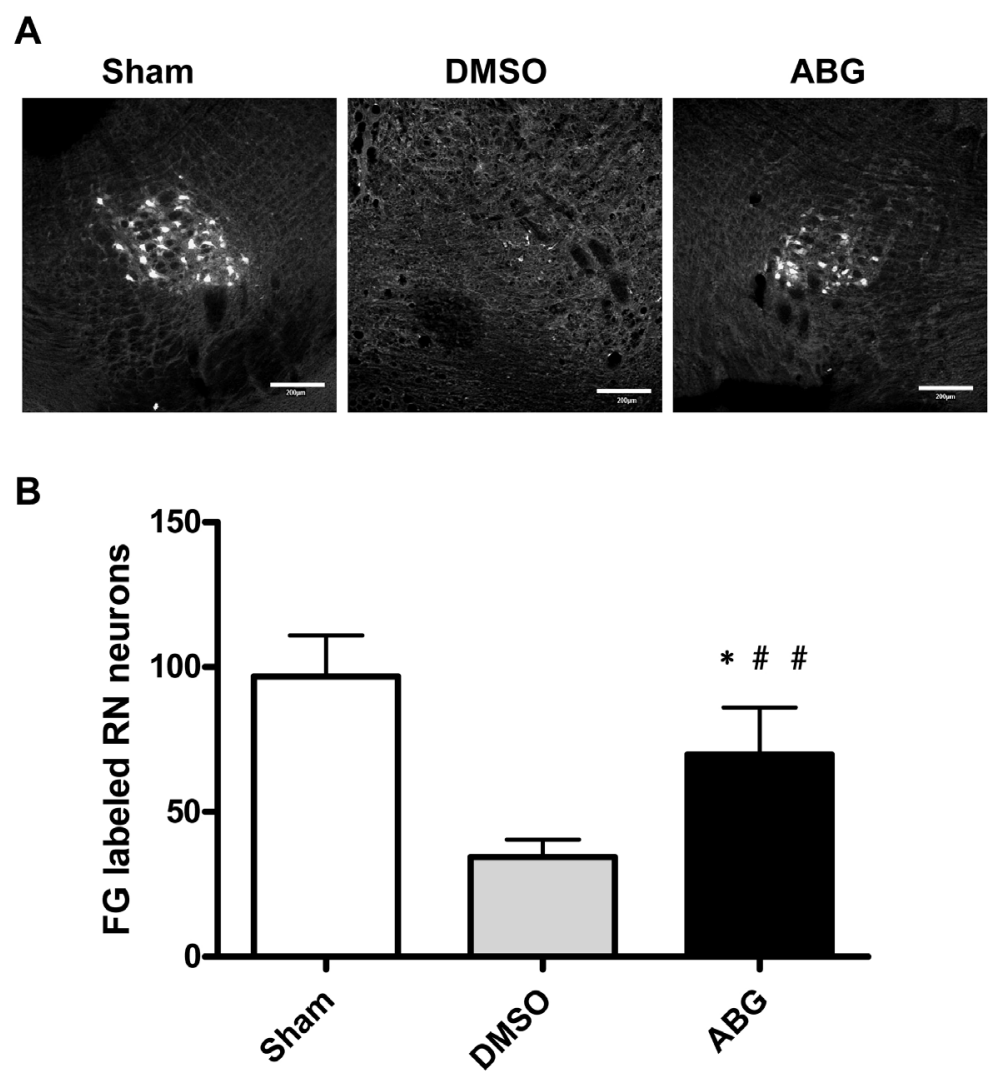

Figure 4. Number of neurons in red nucleus retrogradely labeled with FG injected $5 \mathrm{~mm}$ caudal to the spinal cord lesion site. A. Photomicrographs of midbrain showing FG retrograde tracing of RN neurons. B. Quantification of the FG-labeled neuron numbers of the RN. FG-labeled RN neurons of the SCI rats were significantly decreased compared with the sham group $(\# \mathrm{P}<0.01 . \mathrm{N}=6$ ). The $\mathrm{FG}$ positive neurons were significantly greater in number in $\mathrm{ABG}$-treated rats than in DMSO control rats $\left({ }^{*} \mathrm{P}<0.05, \mathrm{~N}=6\right)$. Data are reported as means $\pm \mathrm{SD}$. Intergroup comparisons were conducted by one-way ANOVA followed by the post hoc test.

with target cells is the goal for repairing SCI and the basis of the functional recovery of SCI patients. Neurotrophic factors are a family of proteins that are not only essential for neuronal differentiation, growth, and survival, but also play an important role in the repair of the central nervous system (Kadoya et al., 2009). A decreased release of neurotrophic factors has been found to impede axonal regeneration after SCI. Exogenous neurotrophic factors can reduce neuronal damage and promote axonal regeneration and functional recovery. The most commonly studied neurotrophic factors include nerve growth factor (NGF), neurotrophin 3 (NT-3), brain-derived neurotrophic factor (BDNF), and glial cell line-derived neurotrophic factor (GDNF), which can respectively act on different types of neurons to promote axonal regeneration. For example, NGF stimulates the regeneration of the sensory conductive pathway and the survival of sensory neurons, NT-3 acts upon corticospinal neurons and promotes the regeneration of the corticospinal tract, and GDNF is the neurotrophic factor of motor neurons 
that promotes survival of corticospinal motor neurons (Tuszynski et al., 2003; Taylor et al., 2004; Ruitenberg et al., 2005). However, there are some difficulties in using neurotrophic factor in the treatment of SCI, such as low stability and short half-life in plasma, and the fact that it can neither be taken orally nor can it pass through the blood-brain barrier (Gao et al., 2010a,b). Although spinal cord microinjection combined with cell transplantation can improve the stability of the exogenous neurotrophic factors (Lu and Tuszynski, 2008), the injection itself may cause a secondary insult in the spinal cord of patients (Kadoya et al., 2009). Fortunately, there are some small molecules in nature that simulate the function of neurotrophic factors or regulate the same signal pathways, which may benefit spinal cord injury and other CNS injuries, as well as overcome the above disadvantages. They thus have great potential for clinical application.

Gentian extract ABG is a novel group of compounds with similar structures purified from Yunnan gentian by the Qi team. Previous studies have found that like NGF, ABG promotes neurite growth and extension of PC12 cells. Additionally, it is more stable than NGF and more easily passes through the blood-brain barrier (Gao et al., 2010a,b; Luo et al., 2011). Methanol extract of gentian can be separated and purified to obtain 11 dihydroxybenzoic acid ester compound monomers with a similar chemical structure: a hydrophilic dihydroxy benzene ring at one end and a hydrophobic long chain carboxylic acid ester at the other end. The main difference among the 11 compounds is the length of the hydroxyl chain, which varies from 2 to 22 carbon atoms. The length of the hydroxyl chain of ABG001 is 14 carbon atoms, and it is the most effective one among 11 compounds in promoting neurite growth (Luo et al., 2011).

In this experiment, after intraperitoneally administering ABG001 to SCI rats every other day for 3 consecutive weeks, the function of $\mathrm{ABG}$ in the recovery of motor function of hind limbs in SCI rats was studied by behavioral assessments, and its effect on survival and regeneration of injured neurons was investigated by morphological studies. The results showed that after the twelfth day post-operation, the rats that received ABG treatment had a better performance in the BBB score and in the grip strength test than those in the DMSO group. ABG has a certain effect on protection or regeneration of neurons in the injured area to reduce tissue edema, hemorrhage, and necrosis. The result of immunofluorescence staining showed less proliferation of glial cells and more regenerated neurons in the injured area for the rats in the ABG group compared to the DMSO group. Furthermore, we used a fluorogold retrograde tracing study to evaluate the effect of $A B G$ on red nucleus neuron regeneration in the rubrospinal tract. It turned out that the number of FG-positive cells transported reversely to the red nucleus significantly increased with ABG treatment compared with the DMSO control group. This indicates that $\mathrm{ABG}$ promotes axon regeneration of red nucleus neurons.

In conclusion, ABG001 has a certain role in protecting neurons in the injured area of the spinal cord, promoting their regeneration and inhibiting glial cell proliferation. Therefore, it has broad prospects for application in the treatment of spinal cord injury, but further research is required to determine the exact mechanism involved.

\section{ACKNOWLEDGMENTS}

Research supported by the Natural Science Foundation of Zhejiang Province (\#LY14H090002 and \#LY15H250001), the Scientific Research Fund of Zhejiang Provincial Education Department (\#Y201120080), and the Foundation of Zhejiang Provincial Health Department (\#2012KYB065). We gratefully acknowledge the assistance of Dr. Jianhua Qi for offering the tetradecyl 2,3-dihydrobenzaote (ABG001). 


\section{REFERENCES}

Barbour HR, Plant CD, Harvey AR and Plant GW (2013). Tissue sparing, behavioral recovery, supraspinal axonal sparing/ regeneration following sub-acute glial transplantation in a model of spinal cord contusion. BMC Neurosci. 14: 106 http://dx.doi.org/10.1186/1471-2202-14-106.

Basso DM (2004). Behavioral testing after spinal cord injury: congruities, complexities, and controversies. J. Neurotrauma 21: 395-404 http://dx.doi.org/10.1089/089771504323004548.

Ding Y, Zhang R, Zhang K, Lv X, et al. (2013). Nischarin is differentially expressed in rat brain and regulates neuronal migration. PLoS One 8: e54563 http://dx.doi.org/10.1371/journal.pone.0054563.

Gao L, Li J and Qi J (2010a). Gentisides A and B, two new neuritogenic compounds from the traditional Chinese medicine Gentiana rigescens Franch. Bioorg. Med. Chem. 18: 2131-2134 http://dx.doi.org/10.1016/j.bmc.2010.02.004.

Gao L, Xiang L, Luo Y, Wang G, et al. (2010b). Gentisides C-K: nine new neuritogenic compounds from the traditional Chinese medicine Gentiana rigescens Franch. Bioorg. Med. Chem. 18: 6995-7000 http://dx.doi.org/10.1016/j. bmc.2010.08.020.

Hu JZ, Huang JH, Xiao ZM, Li JH, et al. (2013). Tetramethylpyrazine accelerates the function recovery of traumatic spinal cord in rat model by attenuating inflammation. J. Neurol. Sci. 324: $94-99$ http://dx.doi.org/10.1016/j.jns.2012.10.009.

Kadoya K, Tsukada S, Lu P, Coppola G, et al. (2009). Combined intrinsic and extrinsic neuronal mechanisms facilitate bridging axonal regeneration one year after spinal cord injury. Neuron 64: 165-172 http://dx.doi.org/10.1016/j. neuron.2009.09.016.

Kim KT, Nam TK, Park YS, Kim YB, et al. (2011). Neuroprotective effect of anthocyanin on experimental traumatic spinal cord injury. J. Korean Neurosurg. Soc. 49: 205-211 http://dx.doi.org/10.3340/jkns.2011.49.4.205.

Lepore AC, Rauck B, Dejea C, Pardo AC, et al. (2008). Focal transplantation-based astrocyte replacement is neuroprotective in a model of motor neuron disease. Nat. Neurosci. 11: 1294-1301 http://dx.doi.org/10.1038/nn.2210.

Liu W, Ding Y, Zhang X and Wang L (2013). Bone marrow stromal cells inhibit caspase-12 expression in rats with spinal cord injury. Exp. Ther. Med. 6: 671-674 10.3892/etm.2013.1201.

Lu P and Tuszynski MH (2008). Growth factors and combinatorial therapies for CNS regeneration. Exp. Neurol. 209: 313-320 http://dx.doi.org/10.1016/j.expneurol.2007.08.004.

Luo Y, Sun K, Li L, Gao L, et al. (2011). Structure-activity relationships of neuritogenic gentiside derivatives. Chem. Med. Chem. 6: 1986-1989. http://dx.doi.org/10.1002/cmdc. 201100348

Ruitenberg MJ, Levison DB, Lee SV, Verhaagen J, et al. (2005). NT-3 expression from engineered olfactory ensheathing glia promotes spinal sparing and regeneration. Brain 128: 839-853 http://dx.doi.org/10.1093/brain/awh424.

Taylor SJ, McDonald JW, 3rd and Sakiyama-Elbert SE (2004). Controlled release of neurotrophin-3 from fibrin gels for spinal cord injury. J. Control. Release 98: 281-294 http://dx.doi.org/10.1016/j.jconrel.2004.05.003.

Thuret S, Moon LD and Gage FH (2006). Therapeutic interventions after spinal cord injury. Nat. Rev. Neurosci. 7: 628643 http://dx.doi.org/10.1038/nrn1955.

Tuszynski MH, Grill R, Jones LL, Brant A, et al. (2003). NT-3 gene delivery elicits growth of chronically injured corticospinal axons and modestly improves functional deficits after chronic scar resection. Exp. Neurol. 181: 47-56 http://dx.doi.org/10.1016/S0014-4886(02)00055-9.

Zhou L, Lu Z, Li L, Chen L, et al. (2014). Pro-neurogenesis and anti-dementia properties of tetradecyl 2,3-dihydroxybenzoate through TrkA receptor-mediated signalling pathways. Int. J. Neuropsychopharmacol. 17: 1847-1861 http://dx.doi. org/10.1017/S1461145714000558. 